\title{
Dense quark-gluon plasma in strong magnetic fields
}

\author{
R. A. Abramchuk ${ }^{1,2, a}$, M. A. Andreichikov ${ }^{1,2}\left(\mathbb{D}\right.$, Z. V. Khaidukov ${ }^{1,2}$, Yu. A. Simonov ${ }^{2}$ \\ ${ }^{1}$ Moscow Institute of Physics and Technology, 9, Institutskii per., Dolgoprudny, Moscow Region 141700, Russia \\ 2 Institute for Theoretical and Experimental Physics of NRC "Kurchatov Institute", B. Cheremushkinskaya 25, Moscow 117259, Russia
}

Received: 16 August 2019 / Accepted: 6 December 2019 / Published online: 30 December 2019

(C) The Author(s) 2019

\begin{abstract}
A non-perturbative (np) method of Field correlators (FCM) was applied to study QCD at temperatures above the deconfinement transition $\left(1<T / T_{C}<3, T_{c} \sim\right.$ $0.16 \mathrm{GeV}$ ) and nonzero baryon densities (baryon chemical potential $\mu_{B}<0.5 \mathrm{GeV}$ ) in an external uniform magnetic field $\left(e B<0.5 \mathrm{GeV}^{2}\right)$. Within FCM, the np hightemperature dynamics is embodied in the Polyakov loop and in the Debye mass due to the Color-Magnetic confinement. Analytic expressions for quark pressure and magnetic susceptibility were obtained. The expressions were represented as series and in integral form. Magnetic susceptibility was found to increase rapidly with temperature and slowly with density. The results at the zero density limit are in agreement with lattice data.
\end{abstract}

\section{Introduction}

Strong magnetic fields emerge in various areas of physics, e.g. in cosmology $[1,2]$, in non-central heavy-ion collisions [3-7], in neutron stars physics [8]; see [9] for review on strongly interacting matter in magnetic fields.

The influence of a magnetic field (m.f.) on QCD thermodynamics was studied in many model approaches [10-39]. In particular, for the results within the Nambu-Jona-Lasinio (NJL) model and the holographic approach see [13-17,40] and $[41,42]$, respectively.

The principal problem of a strongly interacting system description is its non-perturbative dynamics. In the present paper, we address the problem with the Field Correlator Method [43-47] (see [48] for a recent review). The QCD thermodynamics within FCM was initially developed in [4953] with incorporation of Polyakov loops (for a comparison with lattice data, see $[54,55])$.

Recently, the np QCD thermodynamics description at temperatures above deconfinement within FCM was improved

\footnotetext{
a e-mail: abramchuk@phystech.edu
}

by incorporating the color-magnetic confinement (CMC) [56-60]. CMC produces the effective gluon mass, the Debye screening mass $m_{D}$, that linearly grows with temperature above deconfinement.

With this improvement, a solution to the famous Linde problem was suggested in [57]. A compatible with lattice data $\mathrm{SU}(3)$ thermodynamics description was constructed in $[56,58,59]$. An improved EoS for $n_{f}=2+1$ QCD at nonzero baryon density was derived in [60]. In the zero density limit, the results [56-60] are in good agreement with lattice data [61], using the Debye mass found in [62]. At nonzero density, the absence of the critical point in $(\mu, T)$-plane was demonstrated, which is in agreement with lattice data analysis. In addition, the sound speed in QGP at zero and nonzero density was determined in [63] and [64], respectively. For a summary of FCM results at nonzero density see [65].

The topic of the np dynamics behind the Polyakov loop was suggested in [66].

The important subject of thermodynamics in magnetic field was studied in detail in lattice and analytic calculations in [68-75] at zero baryonic density.

In the present paper, we continue studying QCD thermodynamics in a uniform magnetic field within FCM [7679] applying our methods to nonzero density. We start with the quark contribution to the dense deconfined QCD free energy with $\mathrm{np}$ interaction incorporated by means of CMC and Polyakov loops. Then we replace the quark energy with the relativistic charge energy in a m.f to obtain an expression for the free energy in a m.f. Finally, we derive a EoS for the finite baryon density QGP in a m.f., or expression for the QGP pressure, from the free energy expression; we define the QGP magnetic susceptibility with the quark contribution to the QGP pressure. We check our results in a strong m.f. at the zero density limit by numerical comparison with lattice data.

The paper is organized as follows. In Sect. 2 we outline a dense QGP EoS derivation in the absence of a m.f. In Sect. 3 we generalize the EoS to the case of a nonzero m.f. In Sect. 4 
we calculate the QGP magnetic susceptibility. In Sect. 5 we present a numerical evaluation of our analytical results. Section 6 contains conclusion and discussion.

\section{Quark pressure with Polyakov loops and CMC}

We start with a $f$-flavored quark (the index $f=u, d, s \ldots$ is suppressed unless otherwise stated) free energy in background color and electromagnetic fields $A_{\mu}(x)$ and $A_{\mu}^{(e)}(x)$ (for the gluon contribution to the QGP pressure, see [56])

$$
\begin{aligned}
& \frac{1}{T} F_{q}\left(A, A^{(e)}\right)=-\frac{1}{2} \operatorname{tr} \int_{0}^{\infty} \xi(s) \\
& \frac{d s}{s} d^{4} x \overline{(D z)_{x x}} e^{-K-s m_{q}^{2}}\left\langle W_{\sigma}(C[z])\right\rangle,
\end{aligned}
$$

where $K=\frac{1}{4} \int_{0}^{s}\left(\frac{d z_{\mu}}{d \tau}\right)^{2} d \tau$ is the kinetic kernel (we utilized the Feynman-Fock-Schwinger proper time formalism), $m_{q}$ is the current quark mass, and

$$
\begin{aligned}
W_{\sigma}(C[z])= & P_{F} P_{A} \exp \left(i g \int_{C[z]} A_{\mu} d z_{\mu}+i e \int_{C[z]} A_{\mu}^{(e)} d z_{\mu}\right) \\
& \exp \int_{0}^{s}\left(g \sigma_{\mu \nu} F_{\mu \nu}+e \sigma_{\mu \nu} F_{\mu \nu}^{(e)}\right) d \tau
\end{aligned}
$$

is a path- and surface-ordered Wilson loop; $\langle\ldots\rangle$ denotes the averaging over the stochastic color background field. The integration measure implies periodic boundary conditions ( $\beta=T^{-1}$ is the inverse temperature) on a contour $C[z]$ (for details, see $[56,60]$ )

$$
\begin{aligned}
{\overline{(D z)_{x y}}}_{x y}^{w} & \prod_{m=1}^{n} \frac{d^{4} \Delta z_{k}(m)}{(4 \pi \varepsilon)^{2}} \sum_{n=0, \pm 1, \pm 2}(-)^{n} \\
& \frac{d^{4} p}{(2 \pi)^{4}} e^{i p_{\mu}\left(\sum \Delta z_{\mu}(m)-(x-y)-n \beta \delta_{\mu 4}\right)} .
\end{aligned}
$$

We write the series over the Matsubara frequencies in the form

$$
P_{q}=2 N_{c} \int_{0}^{\infty} \frac{d s}{s} e^{-m_{q}^{2} s} \sum_{n=1}^{\infty}(-)^{n+1}\left[S^{(n)}(s)+S^{(-n)}(s)\right],
$$

where

$S^{(n)}(s)=\int(\overline{D z})_{o n}^{w} e^{-K} \frac{1}{N_{c}} \operatorname{tr} W_{\sigma}(C[z])$.

The T-independent $n=0$ term was subtracted.

At this point, we introduce the main np thermodynamics ingredients - CMC and the Polyakov loop $L$. Within the FCM assumptions, color-electric and color-magnetic stochastic background fields are statistically independent. Hence, the Polyakov loop and the spatial $3 \mathrm{~d}$ projection of the quark
Green's function $S_{3}(s)$, which is subjected to CMC, factorize

$$
S^{(n)}(s)=\int\left(D z_{4}\right)_{o n}^{w} e^{-K} L^{n} S_{3}(s)=\frac{e^{\frac{-n^{2} \beta^{2}}{4 s}} L^{n}}{\sqrt{4 \pi s}} S_{3}(s) .
$$

We introduced the quark density by replacing the free energy in (1) with the thermodynamic potential $\Omega=F-\mu N$ ( $\mu$ is the f-flavored quark chemical potential). The pressure definition is $P=-\left(\frac{\partial \Omega}{\partial V}\right)_{T, \mu}$, so

$P_{q}=\frac{4 N_{c}}{\sqrt{4 \pi}} \int_{0}^{\infty} \frac{d s}{s^{3 / 2}} e^{-m_{q}^{2} s} S_{3}(s) \sum_{n=1,2, \ldots}(-)^{n+1} e^{-\frac{n^{2}}{4 T^{2} s}} \cosh \left(\frac{\mu n}{T}\right) L^{n}$,

We may extract the quark Polyakov loop $L=\exp$ $\left(-\frac{V_{1}(\infty, T)}{2 T}\right)$ from lattice simulations (in which case, we may have to change its normalization) or calculate it within FCM [66] (in which case, we still indirectly use lattice data on deconfinement dynamics).

Following $[56,60]$, we utilize the approximation

$$
S_{3}(s) \simeq \frac{1}{(4 \pi s)^{3 / 2}} e^{-\frac{m_{D}^{2} s}{4}}, \quad m_{D}^{2}=c_{D}^{2} \sigma_{s}(T),
$$

where $c_{D} \sim 2$ [62] is a free parameter in our framework, and the color-magnetic string tension $\sigma_{s}(T) \sim g^{4} T^{2}$ calculated in [62] defines magnetic Debye screening mass $m_{D}$.

Therefore, the EoS is

$\frac{P_{q}}{T^{4}}=\frac{N_{c}}{4 \pi^{2}} \sum_{n=1}^{\infty} \frac{(-)^{n+1}}{n^{4}} L^{n} \cosh \left(\frac{\mu n}{T}\right) \Phi_{n}(T)$,

where $K_{2}$ is the Macdonald function

$\Phi_{n}(T)=\frac{8 n^{2} \bar{M}^{2}}{T^{2}} K_{2}\left(\frac{\bar{M} n}{T}\right), \quad \bar{M}=\sqrt{m_{q}^{2}+\frac{m_{D}^{2}}{4}}$.

To reveal the EoS analytical structure in the complex $\mu$ plane, we cast the series to the integral form following [60]

$$
\begin{aligned}
\frac{P_{q}}{T^{4}} & =\frac{N_{c}}{\pi^{2}}\left(\xi_{+}+\xi_{-}\right) \\
\xi_{ \pm} & =\sum_{n=1}^{\infty} \frac{(-)^{n+1}}{n^{2}} L^{n} e^{ \pm \mu n \beta}\left(\frac{\bar{M}}{T}\right)^{2} K_{2}\left(\frac{\bar{M} n}{T}\right) \\
& =\frac{1}{12}\left(\frac{\bar{M}}{T}\right)^{4} \int_{0}^{\infty} \frac{u^{4} d u}{\sqrt{1+u^{2}}} \frac{1}{1+\exp \left(\frac{\bar{M}}{T} \sqrt{1+u^{2}}+\frac{V_{1}}{2 T} \mp \frac{\mu}{T}\right)} .
\end{aligned}
$$

Cuts in the complex plane immediately follow from (13): $\frac{|\operatorname{Re} \mu|}{T} \geq \frac{\bar{M}}{T}+\frac{V_{1}}{2 T}, \frac{\operatorname{Im} \mu}{T}=\pi(2 n+1)$.

In what follows, we also use another form [78] of EoS (9) 


$$
\begin{aligned}
P_{q}= & \frac{2 N_{c}}{\sqrt{\pi}} \int \frac{d^{3} p}{(2 \pi)^{3}} \sum_{n=1}^{\infty}(-)^{n+1} \sqrt{\frac{2}{\beta n}} L^{n} \cosh \left(\frac{\mu n}{T}\right) \int_{0}^{\infty} \\
& \times \frac{d \omega}{\sqrt{\omega}} e^{-\left(\frac{\bar{M}^{2}+\mathrm{p}^{2}}{2 \omega}+\frac{\omega}{2}\right) n \beta} .
\end{aligned}
$$

\section{Quark pressure in a magnetic field}

Let us adjust (14) to the case of a uniform m.f. directed along the $\mathrm{z}$-axis.

The m.f. alters quarks motion in the transverse plane. Transverse motion appears in (14) through the energy spectra and the phase space. To make up for the m.f., we introduce the Landau energy levels and modify the phase space following the standard prescription [80]

$$
\begin{aligned}
& \varepsilon \rightarrow \varepsilon_{n_{\perp}}^{\sigma}\left(p_{z}\right) \\
&=\sqrt{p_{z}^{2}+\bar{M}^{2}+\left|e_{q} B\right|\left(2 n_{\perp}+1-\bar{\sigma}\right)}, \quad \bar{\sigma}=\frac{e_{q}}{\left|e_{q}\right|} \sigma . \\
& 2 \int \frac{V_{3} d^{3} \mathbf{p}}{(2 \pi)^{3}} \rightarrow \sum_{\sigma= \pm 1} \sum_{n_{\perp}=0}^{\infty} \int \frac{d p_{z}}{2 \pi} \frac{\left|e_{q} B\right|}{2 \pi} V_{3} .
\end{aligned}
$$

The standard pressure definition may require an elaboration in case of a non-trivial phase space. However, the quark pressure along the m.f. is well-defined in the present case

$P_{z}=-\left(\frac{\partial \Omega}{\partial V_{z}}\right)_{T, \mu}, \quad d V_{z}=S_{\perp} d z$.

See Sect. 5 for the discussion of pressure anisotropy in magnetized QGP. In what follows, we suppress the subscript, and write $P \equiv P_{z}$.

With this reservation, we obtain

$$
\begin{aligned}
P_{q}(B) & =\sum_{n_{\perp}, \sigma} N_{c} T \frac{\left|e_{q} B\right|}{2 \pi} \frac{\chi(\mu)+\chi(-\mu)}{8}, \\
\chi(\mu) & =\int \frac{d p_{z}}{2 \pi} \ln \left(1+\exp \left(\frac{\mu-V_{1} / 2-\varepsilon_{n_{\perp}}^{\sigma}\left(p_{z}\right)}{T}\right)\right) .
\end{aligned}
$$

The integration yields $\left(\varepsilon_{n_{\perp}}^{\sigma} \equiv \varepsilon_{n_{\perp}}^{\sigma}(0)\right)$

$$
\begin{aligned}
P_{q}(B, T)= & \frac{N_{c}\left|e_{q} B\right| T}{\pi^{2}} \sum_{n_{\perp}, \sigma} \sum_{n=1}^{\infty} \frac{(-)^{n+1}}{n} \\
& \times L^{n} \cosh \left(\frac{\mu n}{T}\right) \varepsilon_{n_{\perp}}^{\sigma} K_{1}\left(\frac{n \varepsilon_{n_{\perp}}^{\sigma}}{T}\right),
\end{aligned}
$$

and the summation of Landau levels over $n_{\perp}$ yields the expression for f-flavored quark pressure at finite density in a m.f. (along the m.f.)

$$
\begin{aligned}
P_{q}(B)= & \frac{N_{c} e_{q} B T}{\pi^{2}} \sum_{n=1}^{\infty} \frac{(-)^{n+1}}{n} L^{n} \cosh \left(\frac{\mu n}{T}\right)\left(\bar{M} K_{1}\left(\frac{n \bar{M}}{T}\right)\right. \\
& +\frac{2 T}{n} \frac{e_{q} B+\bar{M}^{2}}{e_{q} B} K_{2}\left(\frac{n}{T} \sqrt{e_{q} B+\bar{M}^{2}}\right) \\
& \left.-\frac{n e_{q} B}{12 T} K_{0}\left(\frac{n}{T} \sqrt{e_{q} B+\bar{M}^{2}}\right)\right) .
\end{aligned}
$$

The accuracy of the $n_{\perp}$ summation in (20) exemplified in (21) is checked below in Sect. 5 and Fig. 1.

The total quark pressure is the sum over the flavors

$P_{q}^{(t o t)}(B, T)=\sum_{f} P_{q}^{(f)}(B, T)$.

As in the previous section, we cast the series to the integral form (see the Appendix of [78] for details)

$\sum_{n_{\perp}, \sigma} \chi(\mu)=\left(I_{1}+I_{2}+I_{3}\right)$

where

$I_{1}=\frac{1}{\pi T} \int_{0}^{\infty} \frac{p_{z} d p_{z}}{1+\exp \left(\frac{\sqrt{p_{i}^{2}+\bar{M}^{2}}-\mu+V_{1} / 2}{T}\right)}$,

$I_{2}=\frac{1}{\pi T} \int_{0}^{\infty} \frac{p_{z}^{2} d p_{z}}{\left|e_{q} B\right|} \int_{0}^{\infty} \frac{d \lambda}{\sqrt{p_{z}^{2}+\bar{M}^{2}+\left|e_{q} B\right|+\lambda}}$

$$
\begin{gathered}
\frac{1}{1+\exp \left(\frac{\sqrt{p_{z}^{2}+\bar{M}^{2}+\left|e_{q} B\right|+\lambda}-\mu+V_{1} / 2}{T}\right)}, \\
I_{3}=-\frac{\left|e_{q} B\right|}{24 \pi T} \int_{-\infty}^{\infty} \frac{d p_{z}}{\sqrt{p_{z}^{2}+\bar{M}^{2}+\left|e_{q} B\right|}} \frac{1}{1+\exp \left(\frac{\sqrt{p_{z}^{2}+\bar{M}^{2}+\left|e_{q} B\right|}-\mu+V_{1} / 2}{T}\right)} .
\end{gathered}
$$

The integral form of (21) is

$P_{q}(B, T)=N_{c} T \frac{\left|e_{q} B\right|}{2 \pi^{2}} \sum_{i=1}^{3}\left(I_{i}(\mu)+I_{i}(-\mu)\right)$.

Finally, we probe the result (27) with the $B \rightarrow 0$ limit. $I_{1}(\mu)$ does not depend on $B, I_{3}(\mu, B)$ is $O(|B|)$, and $I_{2}(\mu, B)$ is $O\left(|B|^{-1}\right)$, so $B$ drops out

$$
\begin{gathered}
P_{q}=\frac{N_{c} T^{4}}{12 \pi^{2}}\left[\varphi\left(\frac{\mu-\frac{V_{1}}{2}}{T}, \frac{\bar{M}}{T}\right)+\varphi\left(-\frac{\mu+\frac{V_{1}}{2}}{T}, \frac{\bar{M}}{T}\right)\right] \\
\varphi(a, v)=\int_{0}^{\infty} \frac{z^{4} d z}{\sqrt{z^{2}+v^{2}}} \frac{1}{\exp \left(\sqrt{z^{2}+v^{2}}-a\right)+1} .
\end{gathered}
$$

As expected, the expression coincides with the corresponding result (11) of Sect. 2. 


\section{QGP magnetic susceptibility at nonzero baryon density}

We use the following definition of magnetic susceptibility [70] of a thermal medium

$P(B, T)-P(0, T)=\frac{\hat{\chi}}{2}(e B)^{2}+O\left((e B)^{4}\right)$.

The gluon part of the QGP free energy is sensitive to a m.f. via the quark loops. The corresponding contribution to $\hat{\chi}$ is suppressed at least as $\alpha_{s}^{2}$. In the present paper, we neglect this contribution

$\hat{\chi} \simeq \hat{\chi}_{q}^{(t o t)}$.

In a sense, this approximation is similar to the staggered fermions approach.

We also introduce the f-flavored quark susceptibilities $\hat{\chi}_{q}^{(f)}$

$P_{q}^{(f)}(B, T)-P_{q}^{(f)}(0, T)=\frac{\hat{\chi}_{q}^{(f)}}{2}\left(e_{q}^{(f)} B\right)^{2}+O\left((e B)^{4}\right)$,

which are related to the total quark susceptibility as

$\hat{\chi}_{q}^{(t o t)}=\sum_{f} \hat{\chi}_{q}^{(f)}\left(e_{q}^{(f)} / e\right)^{2}$.

We extract the susceptibilities from (21) by expanding it in powers of $e_{q} B$

$\hat{\chi}_{q}=\frac{N_{c}}{3 \pi^{2}} \sum_{n=1}^{\infty}(-)^{n+1} L^{n} \cosh \left(\frac{\mu n}{T}\right) K_{0}\left(\frac{n \bar{M}}{T}\right)$.

With the integral representation for the Macdonald function

$K_{0}(z)=\frac{1}{2} \int_{0}^{\infty} \frac{d x}{x} e^{-\left(\frac{1}{x}+\frac{z^{2} x}{4}\right)}$,

the integral form of the susceptibilities was obtained

$$
\begin{aligned}
\hat{\chi}_{q} & =\frac{N_{c}}{3 \pi^{2}}\left(I_{q}(\mu)+I_{q}(-\mu)\right), \\
I_{q}(\mu) & =\frac{1}{2} \int_{0}^{\infty} \frac{d x}{x} \frac{L e^{(\mu / T)} e^{-\left(\frac{1}{x}+\frac{\bar{M}^{2} x}{4 T^{2}}\right)}}{1+L e^{(\mu / T)} e^{-\left(\frac{1}{x}+\frac{\bar{M}^{2} x}{4 T^{2}}\right)}} .
\end{aligned}
$$

\section{Numerical analysis of the results}

We computed thermodynamic quantities of QGP with u-, d-, s-quarks by means of corresponding series partial summation. The np inputs $V_{1}(\infty, T)$ and $m_{D}(T)$ are identical to these of [65]. They were adjusted in such a way that QGP pressure (9) at zero m.f. and baryon density matches the lattice data $[81,82]$. The Debye mass is defined by the spatial string tension value $m_{D}=c_{D} \sqrt{\sigma_{s}(T)}$; for $\sigma_{s}(T)$ see (45) in [62]. The parameter $c_{D}=1.6$ is close to the corresponding result of [62].

The major source of systematic error is $V_{1}(\infty, T)$ uncertainty. Within the parameters range, we estimate the total error of this section numerical results to be at most $15 \%$ (see Sect. 6 for details).

Let us start with an accuracy check of the approximate Landau levels summation procedure. The Fig. 1 demonstrates the difference between the result of (20) based on the partial sum and the integral form in (21). We find the difference to be negligible in the considered parameter range.

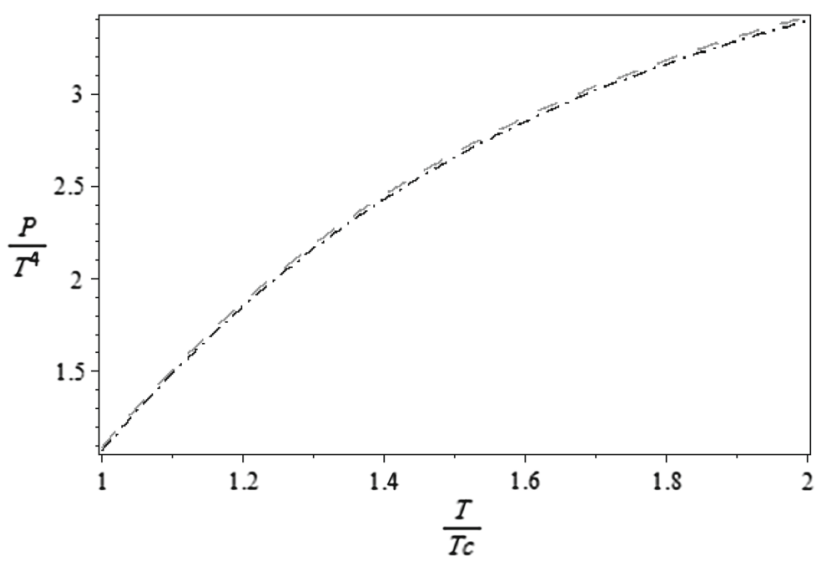

$-\mathrm{eB}=0.2 \mathrm{GeV}^{2}, \mathrm{mu}=0.1 \mathrm{GeV}-\quad-\mathrm{eB}=0.2 \mathrm{GeV}^{2}, \mathrm{mu}=0.1 \mathrm{GeV}$, without resummation

Fig. 1 The pressure $P / T^{4}$ from (20), the dash-dotted line, compared with the integral form, Eq. (21) the dashed line

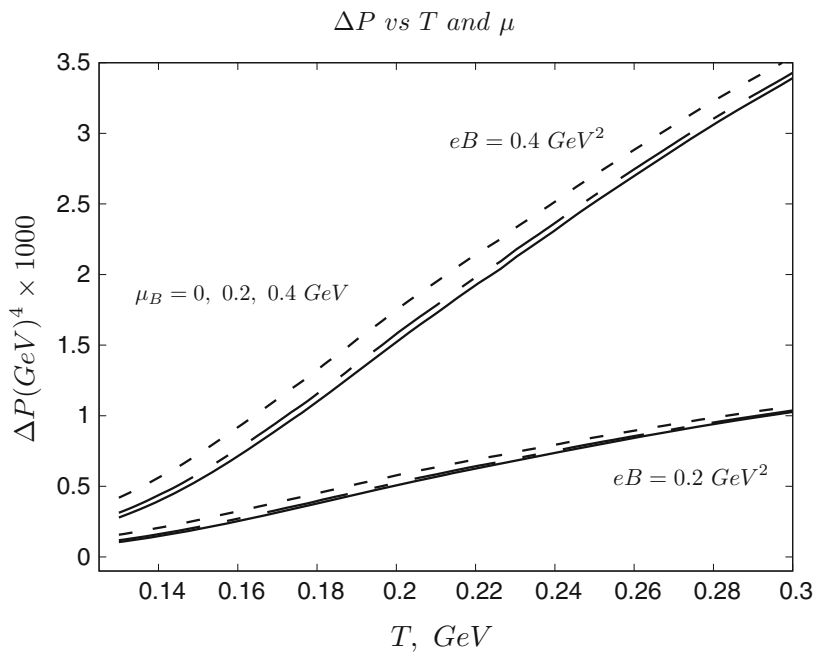

Fig. 2 The magnetic shift $\Delta P=P(e B, \mu)-P(e B=0, \mu)$ for dense QGP with u, d, s quark flavors. For a given m.f., each trajectory demonstrates triple splitting for different $\mu_{B}$. the Solid lines are for $\mu_{B}=0$, the dash-dotted - for $\mu_{B}=0.2 \mathrm{GeV}$, and the dashed - for $\mu_{B}=0.4 \mathrm{GeV}$ 
Fig. 3 The combination $\Delta$ for QGP with u, d, s quark flavors as a function of $T$ at various values of $\mu_{B}[\mathrm{GeV}]$ and $e B\left[\mathrm{GeV}^{2}\right]$

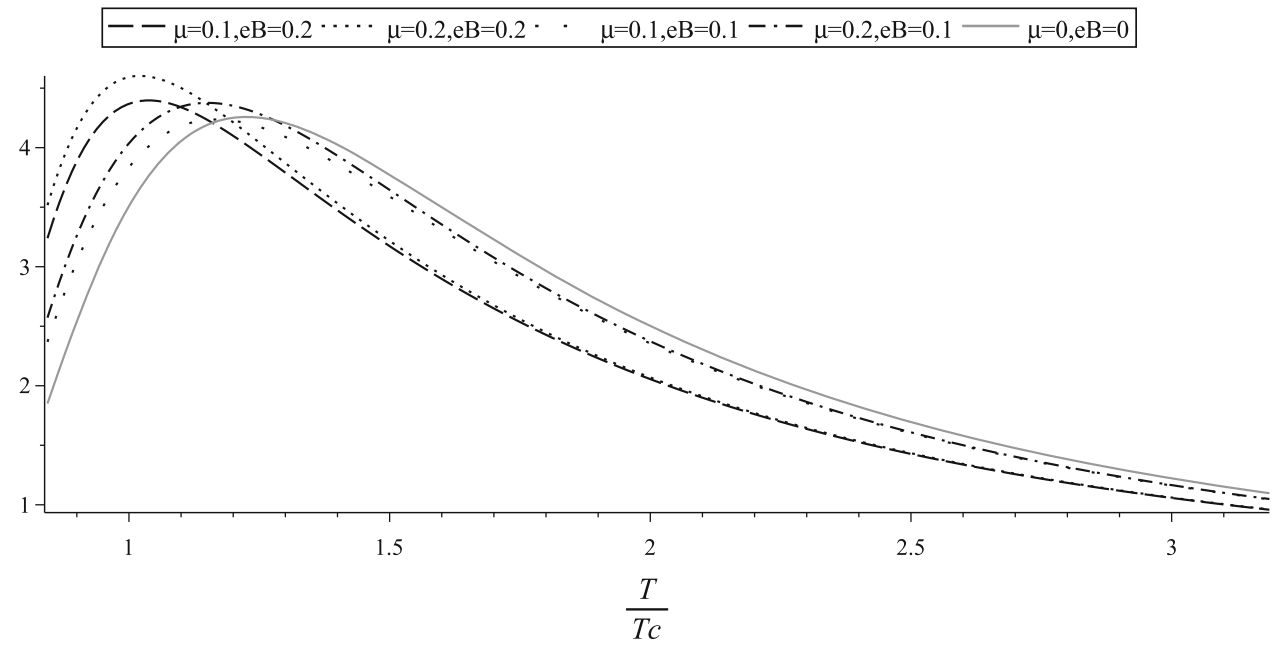

The set of trajectories for the pressure magnetic shift $\Delta P=P\left(e B, \mu_{B}\right)-P\left(0, \mu_{B}\right)$, computed with (21), is presented in Fig. 2 (baryon and quark chemical potentials are related as $\left.\mu_{f}=\frac{1}{3} \mu_{B}, f=u, d, s\right)$. The results suggest strong paramagnetism of QGP, in agreement with the lattice study [69].

With the expressions for gluon pressure from [76] and for quark pressure (21), we computed a special combination $\Delta$ for dense QGP in an external m.f.

$\Delta=\frac{\epsilon-3 P_{z}+\mu n}{T^{4}}$.

If pressure is considered as isotropic, $\Delta$ is the trace anomaly.

The resulting curves in Fig. 3 demonstrate all the qualitative features (the maximum position and width dependence on m.f., the curves intersections) of the same quantity computed with the lattice simulation [73] (see Fig. 12 of [73]) in the given temperature range.

\section{Conclusion and discussion}

In the present paper, pressure of QGP at nonzero baryon density in an external uniform m.f. was calculated analytically for the first time.

The direct influence of a m.f. on the field correlators was studied on the lattice in [83]. Since the effect is at most $10 \%$ at $e B<0.5 \mathrm{GeV}^{2}$, we had assumed it to be negligible for our purposes. The more exact accuracy analysis with the Polyakov loop and the screening mass variation justified this assumption.

Within the parameters range $\left(e B<0.5 \mathrm{GeV}^{2}, \mu_{B}<\right.$ $0.5 \mathrm{GeV}$ ), we neglect the vacuum properties alternation. That is, we neglected the Polyakov loop and the Debye mass dependencies on the baryon density and the m.f. According to the recent lattice data, the approximations $L\left(T, \mu_{B}, B\right) \rightarrow$ $L(T), m_{D}(T, B) \rightarrow m_{D}(T), m_{D}(T, \mu) \rightarrow m_{D}(T)$ yield up to $7 \%$ [84], 5\% [85], and 2\% [86] errors, respectively. We consider the errors as independent, and estimate the total EoS (21) error in "the worst" parameters region (low temperature, high m.f. and density) as $15 \%$. At larger densities, the Polyakov loop dependence on the chemical potential is to be accounted for.

The Polyakov loops interaction and the perturbative corrections were also neglected. The non-perturbative numerical input data were set as to reproduce the correct QGP pressure values at the zero m.f. and baryon density limit.

According to FCM method, spatial string tension $\sigma_{s}(T) \sim$ $M_{D}^{2}$ is defined by the correlator of the color-magnetic gluon fields $\langle B B\rangle$ and the Polyakov loop $L(T) \sim e^{-\frac{V_{1}(\infty)}{T}}$ is defined by the corresponding color-electric correlator $\langle E E\rangle$ (The detailed discussion of the thermal FCM correlators is provided in $[56,60,62])$. However, the $\mathrm{CE}$ and CM correlators demonstrate the dependence on magnetic field through the fluctuating $q \bar{q}$-pairs. These effects were calculated in $[66,67]$ and the total effect lies beyond the total error of $15 \%$ for the MF strengths $\left(e B=0.2,0.4 \mathrm{GeV}^{2}\right)$ used in present calculation.

With the numerical analysis of our results, we observed QGP strong paramagnetism, which was previously found within lattice simulations. The possible pressure anisotropy, on the other hand, is a matter of discussion.

QGP pressure anisotropy is observed in lattice simulations with a certain setup. Basic methods for pressure calculation on lattice are [68]

1. $B=$ const method - the variation of energy in a constant external m.f. (the QGP is assumed to be in thermal equilibrium);

2. $\Phi=$ const method, in which the total magnetic flux $\Phi$ through the lattice is fixed. 
With the first method, pressure is isotropic, while with the second method

$P_{x}=P_{y}=P_{z}-M \cdot B$,

where $M=-\frac{1}{V} \frac{\partial F}{\partial B}$ is the QGP magnetization.

The "anisotropic" method is more subtle for real physical systems. It requires a careful analysis of the energy exchange between longitudinal and transverse motion of quarks and the corresponding relaxation time. We leave these details to a future publication.

Acknowledgements The authors are grateful to B.O. Kerbikov and M.S. Lukashov for useful discussions, and to N.P. Igumnova for technical help in the manuscript preparation. This work was supported by the Russian Science Foundation Grant number 16-12-10414.

Data Availability Statement This manuscript has no associated data or the data will not be deposited. [Authors' comment: This is a theoretical study and no experimental data has been listed.]

Open Access This article is licensed under a Creative Commons Attribution 4.0 International License, which permits use, sharing, adaptation, distribution and reproduction in any medium or format, as long as you give appropriate credit to the original author(s) and the source, provide a link to the Creative Commons licence, and indicate if changes were made. The images or other third party material in this article are included in the article's Creative Commons licence, unless indicated otherwise in a credit line to the material. If material is not included in the article's Creative Commons licence and your intended use is not permitted by statutory regulation or exceeds the permitted use, you will need to obtain permission directly from the copyright holder. To view a copy of this licence, visit http://creativecomm ons.org/licenses/by/4.0/.

Funded by SCOAP ${ }^{3}$.

\section{References}

1. T. Vachaspati, Phys. Lett. B 265, 258 (1991)

2. K. Enqvist, P. Olesen, Phys. Lett. B 319, 178 (1993). arXiv:hep-ph/9308270

3. D.E. Kharzeev, L.D. McLerran, H.J. Warringa, Nucl. Phys. A 803, 227 (2008)

4. V. Skokov, A.Y. Illarionov, V. Toneev, Int. J. Mod. Phys. A 24, 5925 (2009). arXiv:0907.1396

5. V. Voronyuk, V. Toneev, W. Cassing, E. Bratkovskaya, V. Konchakovski et al., Phys. Rev. C 83, 054911 (2011). arXiv:1103.4239

6. A. Bzdak, V. Skokov, Phys. Lett. B 710, 171 (2012). arXiv: 1111.1949

7. W.-T. Deng, X.-G. Huang, Phys. Rev. C 85, 044907 (2012). arXiv: 1201.5108

8. R.C. Duncan, C. Thompson, Astrophys. J. 392, L9 (1992)

9. D.E. Kharzeev, K. Landsteiner, A. Schmitt, H.-U. Yee, Lect. Notes Phys. 871, 1 (2013)

10. T.D. Cohen, D.A. McGady, E.S. Werbos, Phys. Rev. C 76, 055201 (2007). arXiv:0706.3208

11. J.O. Andersen, Phys. Rev. D 86, 025020 (2012). arXiv:1202.2051

12. J.O. Andersen, JHEP 1210, 005 (2012). arXiv: 1205.6978

13. S.P. Klevansky, R.H. Lemmer, Phys. Rev. D 39, 3478 (1989)

14. D .P. Menezes, M. Benghi Pinto, S .S. Avancini, C. Providencia, Phys. Rev. C 80, 065805 (2009). arXiv:0907.2607
15. R. Gatto, M. Ruggieri, Phys. Rev. D 83, 034016 (2011). arXiv: 1012.1291

16. R. Gatto, M. Ruggieri, Phys. Rev. D 82, 054027 (2010). arXiv: 1007.0790

17. K. Kashiwa, Phys. Rev. D 83, 117901 (2011). arXiv:1104.5167

18. J.O. Andersen, R. Khan, Phys. Rev. D 85, 065026 (2012). arXiv: 1105.1290

19. S. S. Avancini, D. P. Menezes, M. B. Pinto, and C. Providencia (2012), arXiv: 1202.5641

20. K. Fukushima and J. M. Pawlowski (2012), arXiv: 1203.4330

21. A.J. Mizher, M.N. Chernodub, E.S. Fraga, Phys. Rev. D 82, 105016 (2010a). arXiv:1004.2712

22. J.O. Andersen, A. Tranberg, JHEP 08, 002 (2012). arXiv: 1204.3360

23. S. Kanemura, H.-T. Sato, H. Tochimura, Nucl. Phys. B 517, 567 (1998). arXiv:hep-ph/9707285

24. K.G. Klimenko, Theor. Math. Phys. 90, 1 (1992)

25. J. Alexandre, K. Farakos, G. Koutsoumbas, Phys. Rev. D 63, 065015 (2001). arXiv:hep-th/0010211

26. D.D. Scherer, H. Gies, Phys. Rev. B 85, 195417 (2012). arXiv: 1201.3746

27. C.V. Johnson, A. Kundu, JHEP 12, 053 (2008). arXiv:0803.0038

28. F. Preis, A. Rebhan, A. Schmitt, JHEP 1103, 033 (2011). arXiv: 1012.4785

29. A.J. Mizher, E.S. Fraga, M. Chernodub, PoS FACESQCD 020, (2010). arXiv:1103.0954

30. J. Gasser, H. Leutwyler, Phys. Lett. B 184, 83 (1987)

31. J. Gasser, H. Leutwyler, Phys. Lett. B 188, 477 (1987b)

32. P. Gerber, H. Leutwyler, Nucl. Phys. B 321, 387 (1989)

33. N. Agasian, S. Fedorov, Phys. Lett. B 663, 445 (2008). arXiv:0803.3156

34. E.S. Fraga, A.J. Mizher, Nucl. Phys. A 820, 1030 (2009). arXiv:0810.3693

35. E.S. Fraga, L.F. Palhares, Phys. Rev. D 86, 016008 (2012). arXiv: 1201.5881

36. E. S. Fraga, arXiv:1208.0917, in: Lect. Notes Phys. (Springer)

37. R. Gatto and M. Rugieri, arXiv:1207.3190, in: Lect. Notes Phys. (Springer)

38. G. Bali, F. Bruckmann, G. Endrodi et al., JHEP 1202, 044 (2012). arXiv: 1111.4956

39. G.S. Bali, F. Bruckmann, G. Endródi, Z. Fodor et al., Phys. Rev. D 86, 071502 (2012). arXiv: 1206.4205

40. A. Ayala, L.A. Hernandez, M. Loewe, A. Raya, J.C. Rojas, R. Zamora, Phys. Rev. D 96, 034007 (2017)

41. J.F. Fuiri III, L.G. Yaffe, JHEP 1507, 116 (2015)

42. R. Oritelli, R. Rougemont, S.I. Finazzo, J. Noronha, Phys. Rev. D 94, 125019 (2016)

43. H.G. Dosh, Phys. Lett. B 190, 177 (1987)

44. H.G. Dosh, YuA Simonov, Phys. Lett. B 205, 339 (1988)

45. YuA Simonov, Nucl. Phys. B 307, 512 (1988)

46. A. Di Giacomo, H.G. Dosch, V.I. Shevchenko, YuA Simonov, Phys. Rept. 372, 319 (2002). hep-ph/0007223

47. YuA Simonov, Phys. Usp. 39, 313 (1996)

48. YuA Simonov, Phys. Rev. D 99, 056012 (2019). arXiv: 1804.08946

49. YuA Simonov, JETP Lett. 54, 249 (1991)

50. YuA Simonov, JETP Lett. 55, 605 (1992)

51. YuA Simonov, Phys. At. Nucl. 58, 309 (1995)

52. Yu. A. Simonov, Proc. Varenna 1995, Selected Topics in Nonperturbative QCD, p. 319

53. H.G. Dosch, H.-J. Pirner, YuA Simonov, Phys. Lett. B 349, 335 (1993)

54. YuA Simonov, Ann. Phys. (NY) 323, 783 (2008)

55. E.V. Komarov, YuA Simonov, Ann. Phys. (NY) 323, 1230 (2008). arXiv:0707.0781

56. N.O. Agasian, M.S. Lukashov, YuA Simonov, Eur. Phys. J. A 53, 138 (2017). arXiv:1701.07959 
57. YuA Simonov, Phys. Rev. D 96, 096002 (2017). arXiv: 1605.07060

58. M.S. Lukashov, YuA Simonov, JETP Lett. 105, 659 (2017). arXiv:1703.06666 [hep-ph]

59. N.O. Agasian, M.S. Lukashov, YuA Simonov, Mod. Phys. Lett. A 31, 1050222 (2016). arXiv:1610.01472 [hep-lat]

60. M.A. Andreichikov, M.S. Lukashov, YuA Simonov, Int. J. Mod. Phys. A 33, 8 (2018). arXiv:1707.04631

61. S.Z. Borsonyi et al., J. High Energy Phys. 2010, 077 (2010). arXiv: 1007.2580

62. N.O. Agasian, YuA Simonov, Phys. Lett. B 639, 82 (2006). hep$\mathrm{ph} / 0604004$

63. Z.V. Khaidukov, YuA Simonov, Phys. Rev. D 98, 074031 (2018). arXiv: 1806.09407

64. Z.V. Khaidukov, YuA Simonov,. arXiv:1811.08970

65. Z.V. Khaidukov and Y.A. Simonov, Phys. Rev. D 100, 076009 , arXiv: 1906.08677

66. R.A. Abramchuk, Z.V. Khaidukov, Y.A. Simonov, arXiv: 1812.01998

67. YuA Simonov, M.A. Trusov, Phys. Lett. B 747, 48 (2015). arXiv:1503.08531

68. G.S. Bali, F. Bruckmann, G. Endrodi, F. Gruber, A. Schafer, JHEP 2013, 130 (2013). arXiv: 1303.1328

69. C. Bonati, M. D’Elia, M. Mariti, F. Negro, F. Sanfilippo, Phys. Rev. Lett. 111, 182001 (2013). arXiv:1307.8063

70. C. Bonati, M. D'Elia, M. Mariti, F. Negro, F. Sanfilippo, 31th International Symposium Lattice 2013, arXiv:1312.5070

71. C. Bonati, M. D’Elia, M. Mariti, F. Negro, F. Sanfilippo, Phys. Rev. D 89, 054506 (2014). arXiv: 1310.8656

72. G.S. Bali, F. Bruckmann, G. Endrodi, F. Gruber, A. Schafer, 31th International Symposium Lattice 2013, arXiv:1310.8145
73. G.S. Bali, F. Bruckmann, G. Endrodi, S.D. Katz, A. Schafer, JHEP 08, 177 (2014). arXiv: 1406.0269

74. M. Strickland, V. Dexheimer, D.P. Menezes, Phys. Rev. D 86, 125032 (2012). arXiv: 1209.3276

75. A.Y. Potekhin, D.G. Yakovlev, Phys. Rev. C 85, 039801 (2012). arXiv: 1109.3783

76. V.D. Orlovsky, YuA Simonov, JETP Lett. 101, 423 (2015). arXiv:1405.2697

77. V.D. Orlovsky, YuA Simonov, IJMPA 30, 155060 (2015) arXiv: 1406.1056

78. V.D. Orlovsky, YuA Simonov, Phys. Rev. D 89, 074034 (2014). arXiv: 1311.1087

79. M.A. Andreichikov, YuA Simonov, Eur. Phys. J. C 78, 420 (2018). arXiv: 1712.02925

80. L.D. Landau, E.M. Lifshitz, Statistical Mechaniscs, Part I, vol. 5 (Pergamon, New York, 1980)

81. S. Borsanyi, Z. Fodor, C. Hoelbling, Phys. Lett. B 370, 99-104 (2014). arXiv:1309.5258 [hep-lat]

82. A.Bazavov, T.Bhattacharya, C. DeTar et al., Phys. Rev. D 90 , 094503, arXiv:1407.6387

83. M. D’Elia, E. Meggiolaro, M. Mesiti, F. Negro, Phys. Rev. D 93 054017 (2016). arXiv: 1510.07012

84. V.V. Braguta, M.N. Chernodub, AYu. Kotov, A.V. Molochkov, A.A. Nikolaev, arXiv:1909.09547

85. C. Bonati, M. D’Elia, M. Mariti, M. Mesiti, F. Negro, A. Rucci, F. Sanfilippo, arXiv:1703.00842

86. M. D’Elia, F. Negro, A. Rucci, F. Sanfilippo, arXiv:1907.09461 